\title{
Servicios de agua potable, saneamiento básico y problemas de salud asociados al consumo hídrico en el cantón Quevedo, Ecuador
}

\author{
María Lorena Cadme Arévalo \\ mcadme@uteq.edu.ec \\ Universidad Técnica Estatal de Quevedo \\ Avenida Quito km 1.5 vía a Santo Domingo de los Tsáchila \\ Quevedo, Los Ríos, Ecuador C.P. 120501 \\ Loguard Smith Rojas Uribe \\ Universidad Técnica Estatal de Quevedo \\ Avenida Quito km 1.5 vía a Santo Domingo de los Tsáchila, \\ Quevedo, Los Ríos, Ecuador C.P. 120501 \\ Thais Sarah Arreaga Cadme \\ Universidad de Barcelona. \\ Gran Vía de las Cortes Catalanas 585 Barcelona, \\ Cataluña, España \\ Ángel Virgilio Cedeño Moreira \\ Universidad Técnica Estatal de Quevedo \\ Avenida Quito km 1.5 vía a Santo Domingo de los Tsáchila, \\ Quevedo, Los Ríos, Ecuador C.P. 120501 \\ Betty Beatriz González Osorio \\ Universidad Técnica Estatal de Quevedo. \\ Avenida Quito km 1.5 vía a Santo Domingo de los Tsáchila, \\ Quevedo, Los Ríos, Ecuador C.P. 120501 \\ Luis Alejandro Saltos Velasquez \\ Universidad Técnica Estatal de Quevedo \\ Avenida Quito km 1.5 vía a Santo Domingo de los Tsáchila \\ Quevedo, Los Ríos, Ecuador C.P. 120501
}

\section{RESUMEN}

Los servicios de saneamiento básico y acceso agua potables es una de las vías para que la vida de los hogares sea la saludable. Por lo tanto, el presente estudio tuvo como objetivo identificar los servicios de agua potable, saneamiento básico y problemas de salud que tienen los hogares del cantón Quevedo por el consumo hídrico. El trabajo fue de característica cualitativa y cuantitativa, con el fin de encontrar resultados confiables y útiles inducidos por la propia comunidad para beneficiar su situación colectiva. La encuesta fue la técnica que se empleó para la recolección de datos para medir las variables 
bajo estudio, se realizó una prueba piloto esta permitió hacer ajustes necesarios y se identificaron limitaciones. La información recolectada fue ejecutada por los investigadores para la construcción de la base de datos propia usando Excel. Los resultados encontrados demostraron que el 59, $4 \%$ de los hogares tienen acceso agua potable, el 36,5 y 16,7 \% de las viviendas adquieren agua con mal sabor y olor. También, se encontró que en el 55,9 \% de los hogares sus habitantes han presentado síntomas de diarreas por el consumo de agua, seguido de 13,8; 9,1 y 8,8 \%. de hogares con vómitos, cólicos y dolores abdominales.

Palabras claves: acceso al agua; salud; contaminación; técnica de purificación del agua. 


\title{
Serviços de água potável, saneamento básico e problemas de saúde associados ao consumo de água no cantão de Quevedo, Equador
}

\begin{abstract}
Basic sanitation services and access to drinking water is one of the ways for household life to be healthy. Therefore, the present study aimed to identify the drinking water services, basic sanitation and health problems that households in the Quevedo canton have due to water consumption. The work was qualitative and quantitative, in order to find reliable and useful results induced by the community itself to benefit their collective situation. The survey was the technique used to collect data to measure the variables under study, a pilot test was carried out, which allowed the necessary adjustments to be made and limitations were identified. The information collected was executed by the researchers for the construction of their own database using Excel. The results found showed that $59.4 \%$ of households have access to drinking water, 36.5 and $16.7 \%$ of households purchase water with a bad taste and smell. Also, it was found that in $55.9 \%$ of the homes its inhabitants have presented symptoms of diarrhea due to the consumption of water, followed by $13.8 ; 9.1$ and $8.8 \%$. from homes with vomiting, colic and abdominal pain.
\end{abstract}

Keywords: access to water; health; pollution; water purification technique.

Artículo recibido: 10 Setiembre. 2021 Aceptado para publicación: 15 Octubre. 2021

Correspondencia: mcadme@uteq.edu.ec Conflictos de Interés: Ninguna que declarar 


\section{INTRODUCCIÓN}

El agua es un insumo necesario para la vida, tiene un alto nivel de importancia en la sociedad debido a que lo abarca todo (Castro et al., 2014). En este sentido, el recurso hídrico influye directamente en los sectores económicos, incluido y especialmente agricultura, generación de energía y manufactura (Mahlknecht, y Pastén, 2013). En base al consumo humano la importancia del acceso al agua potable y a las instalaciones mejoradas de saneamiento ha impulsado en general la agenda mundial del agua, saneamiento e higiene (Dalin et al., 2012). Sin embargo, un gran segmento de la población mundial, especialmente en los países en desarrollo, sigue sin acceso (CEPAL, 2017). El saneamiento y acceso agua potable de forma segura es la ruta para que la vida de los pueblos sea saludable para sus habitantes (Fernández, 2012).

En materia de saneamiento, la región de América Latina y el Caribe (ALC) está por detrás de otras regiones en todo el mundo. En 2015, solo el 22\% de la población de ALC utilizó un servicio de saneamiento administrado donde las excretas se eliminaban de manera segura en in situ o tratado fuera del sitio. En comparación, a nivel mundial, este indicador fue del 39\% (FAO, 2018). Alrededor del 22\% de la población de ALC utilizaba instalaciones de saneamiento conectadas a alcantarillas de las que las aguas residuales recibió tratamiento, y el $85 \%$ de la población utilizó al menos un servicio de saneamiento básico. Esto significa que 222 millones de personas en la región de ALC aún carecía incluso de un servicio de saneamiento básico, y en su lugar utilizaba instalaciones mejoradas compartidas, letrinas de pozo o defecación al aire libre (FAO, 2018). Esto se encuentra relacionado con la ubicación geográfica y la economía de las localidades, siendo los hogares situados ruralmente los mas vulnerables al acceso de servicio básicos (García, 2015). Sin embargo, el crecimiento poblacional ha influido a que los hogares ubicados en el área urbana carezcan de agua potable y saneamiento básico (CIPAL, 2017). Es por ello, que el presente estudio tuvo como objetivo identificar los servicios de agua potable, saneamiento básico y problemas de salud que tienen los hogares del cantón Quevedo por el consumo hídrico.

\section{METODOLOGÍA}

\section{Diseño de estudio}

El presente estudio fue participativo que tuvo una característica cuanti-cualitativo, con el 
fin de encontrar resultados fiables y útiles inducidos por la propia comunidad para beneficiar su situación colectiva. Estos resultados se enfocaron a las dificultades identificadas sobre el acceso al agua y tipo de saneamiento en hogares de tres localidades del cantón Quevedo, específicamente en las parroquias San Carlos, La Esperanza y Quevedo.

\section{Procedimiento de recolección de datos}

La encuesta fue la técnica que se empleó para la recolección de datos para medir las variables bajo estudio, se realizó una prueba piloto esta permitió hacer ajustes necesarios y se identificaron limitaciones. La información recolectada fue ejecutada por los investigadores para la construcción de la base de datos propia usando Excel. Lo que posteriormente fueron analizados empleando técnicas de la estadística descriptiva. Las variables descritas son: acceso al agua, calidad de agua en domicilio, rutas de excretas, problemas de pozos sépticos, síntomas de enfermedades relacionadas al consumo del agua, técnica de purificación del agua y conocimiento de carbón activado.

\section{RESULTADOS Y DISCUSIÓN}

La figura 1a se muestra cual es el abastecimiento de agua que tienen los hogares del cantón Quevedo, se observa que el 59,40 \% de las viviendas tienen acceso a agua potable. Estos resultados nos indica que la mayor parte de la población posee el mejor beneficio de adquisición hídrica. De igual manera, el Instituto Nacional de Estadistas y Censo (INEC, 2017) reporto que el 70,1\% de los hogares en Ecuador tiene acceso a agua segura, pero de forma desigual y este factor también depende de la ubicación geográfica donde se encuentran las viviendas. En este sentido, Ramírez et al. (2015) sostienen que el agua potable se produce a bajo costo, pero la mayoría de comunidades que se encuentra en países subdesarrollos no cuenta con rubros económicos para la construcción de tecnologías de purificación de agua; lo cual, constituye un riesgo alto para la salud de las personas (Domínguez, 2010).

En la figura $1 \mathrm{~b}$ se visualiza la calidad de agua en domicilios, se observa que un 36,5 y $16,7 \%$ obtiene agua con mal sabor y olor, estos son los mayores problemas que enfrentan los hogares. Lo cual está asociado a reacciones de productos químicos que se emplean como tratamiento en el sistema de distribución, por ejemplo, el cloro cuando reacciona con sustancias orgánicas causa problemas de sabor y olor (Bracho-Fernández y 
Fernández-Rodríguez, 2017). En ocasiones los umbrales de cloruros pueden excederse, así generando un sabor salado.

Respecto a la variable servicio de desagüe que tiene los hogares del cantón Quevedo, se encontró que el 84,5\% posee fosa séptica y el otro $15,5 \%$ tiene desagüe municipal (figura 1c). Esto se lo traduce a que toda la población del municipio de Quevedo tiene un sistema de eliminación de excretas. Resultados que se relacionan con lo referido por el INEC (2016), quien indica que un 85,9\% de las viviendas en Ecuador cuenta con un sistema de saneamiento básico. Sin embargo, se encontró que el 27,3 \% de los hogares del cantón Quevedo con pozos sépticos, sus habitantes presentan diarreas por esta ruta de eliminación de excretas, a su vez, se detectó que el 26,5 \% de viviendas tienen problemas con cucarachas, a esto le acompaña un $19,4 \%$ de domicilios que son afectados por los malos olores (figura 1d). Estos resultados demuestran que los domicilios con fosas sépticas son los que presentan el mayor riesgo a enfermedades infecciosas asociadas a las excretas (García et al., 2015 y Guzmán et al., 2015).

Figura 1. Resultados de la encuesta sobre el acceso a agua (a), calidad del agua en domicilio (b), rutas de excretas (c) y problemas de pozos sépticos (d) del cantón Quevedo, provincia Los ríos, Ecuador.
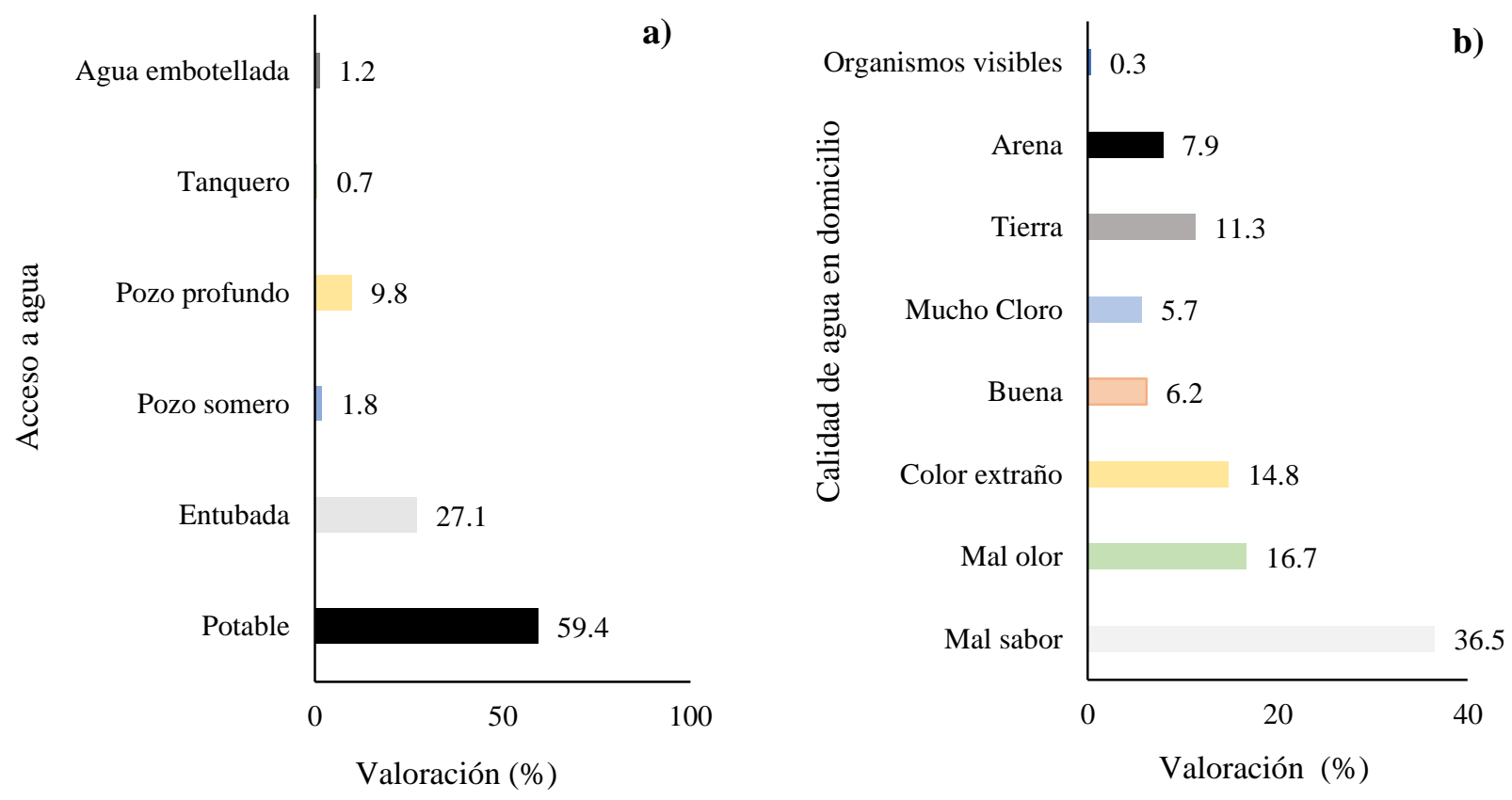

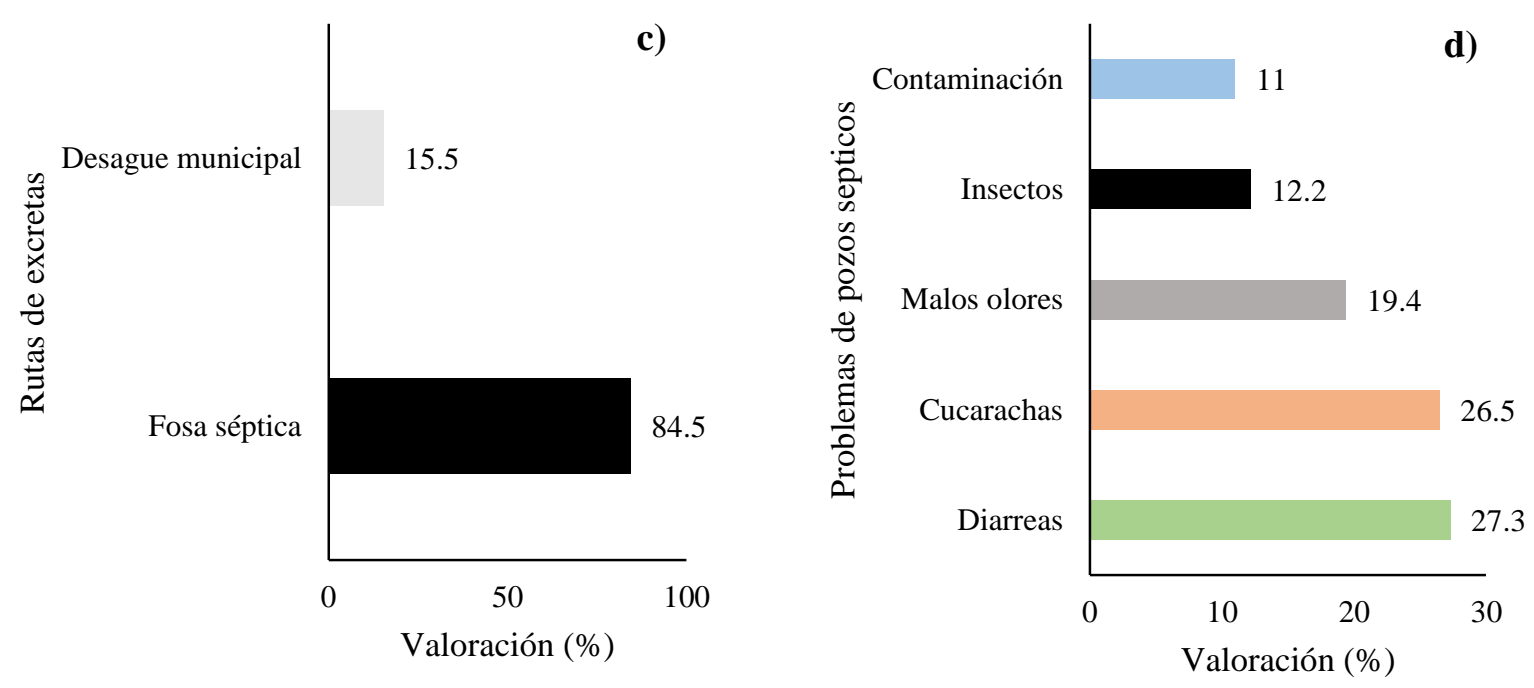

En el 55,90 \% de los hogares del cantón Quevedo se presenta síntomas de diarreas por el consumo de agua, seguido por malestares de vomito $(13,8 \%)$, cólicos $(9,1 \%)$ y dolor abdominal (8,8 \%). Estos fenómenos coinciden con un estudio realizado por Brossard et al. (2020) sobre enfermedades de transmisión hídricas en el cantón Penipe, Ecuador, donde refieren que las enfermedades relacionadas con el agua, son causadas por microorganismos y sustancias químicas, factores que provocan daños gastrointestinales. Resultados que se encuentran estrechamente relacionado con lo manifestado por el Instituto Nacional de Estadísticas y Censo, quien ha descrito que aproximadamente el 20,7 \% del agua destinada al consumo humano en Ecuador está contaminada con heces fecales, esto influye a que la población presente daños en el sistema digestivo (INEC, 2017). Sin embargo, el ministerio de salud pública manifiesta que la provincia de Los Ríos presenta uno de los menores índice de enfermedades trasmitidas por el agua (Ministerio de Salud, 2021), estado en el que se encuentra el municipio de Quevedo.

Figura 2. Resultados de la encuesta sobre síntomas de enfermedades relacionados al consumo del agua del cantón Quevedo, provincia Los ríos, Ecuador.

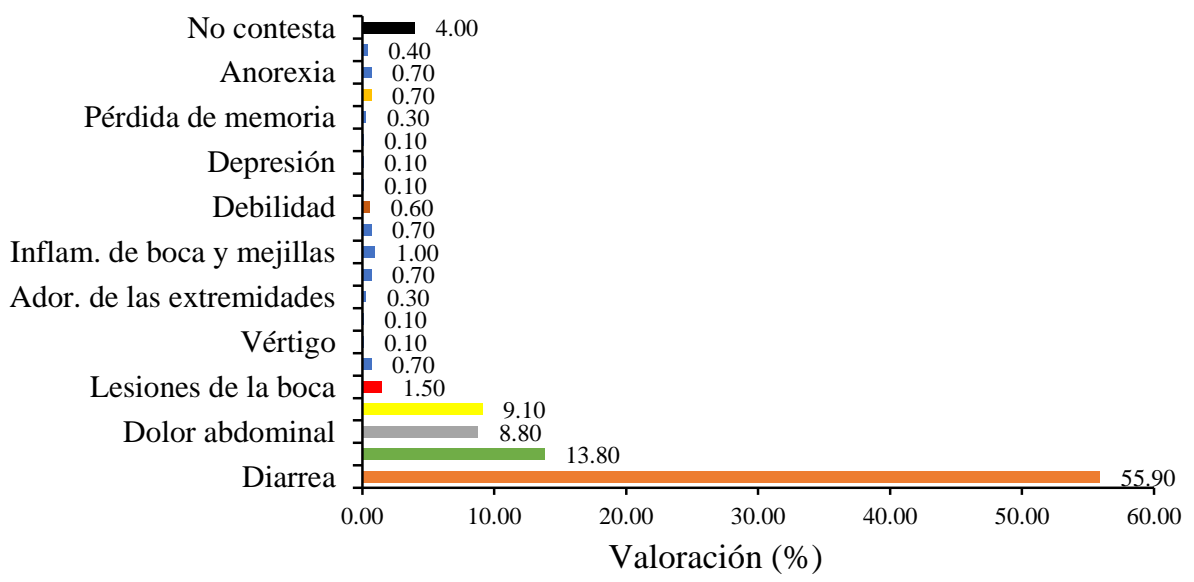


En la figura 3a se presenta la técnica de purificación del agua que tienen los hogares, se observa que el 66,9 \% de los hogares previamente al consumo hierve el agua, un 25,5\% usa el método de cloración, alrededor del 2,1 \% realiza purificación doméstica y aproximadamente un 1,90 \% efectúa la técnica por embotellación. Estos resultados demuestran que en los hogares del cantón Quevedo no se utiliza ningún tipo de método para remover sustancias toxicas, como son los metales pesados. El carbón activado es una de las técnicas que se emplea para eliminar estas moléculas que producen daños a la salud. A todo esto, se le suma que el 82,2 \% de los hogares no conoce que es el carbón activado (figura 3b) y que beneficios tiene esta técnica. Lo cual podría ser también uno de las causas de los problemas gastrointestinales que presentan los habitantes del municipio de Quevedo (figura 2). Debido a que un estudio realizado por Mite et al. (2010), se encontró que las fuentes hídricas de la provincia de los Ríos se encuentran contaminadas por cadmio, metal pesado que causa daños digestivos, cardiacos y renales.

Figura 3. Resultados de la encuesta sobre la técnica de purificación del agua (a) y el conocimiento del carbón activado (b) en las viviendas del cantón Quevedo, Los Ríos, Ecuador

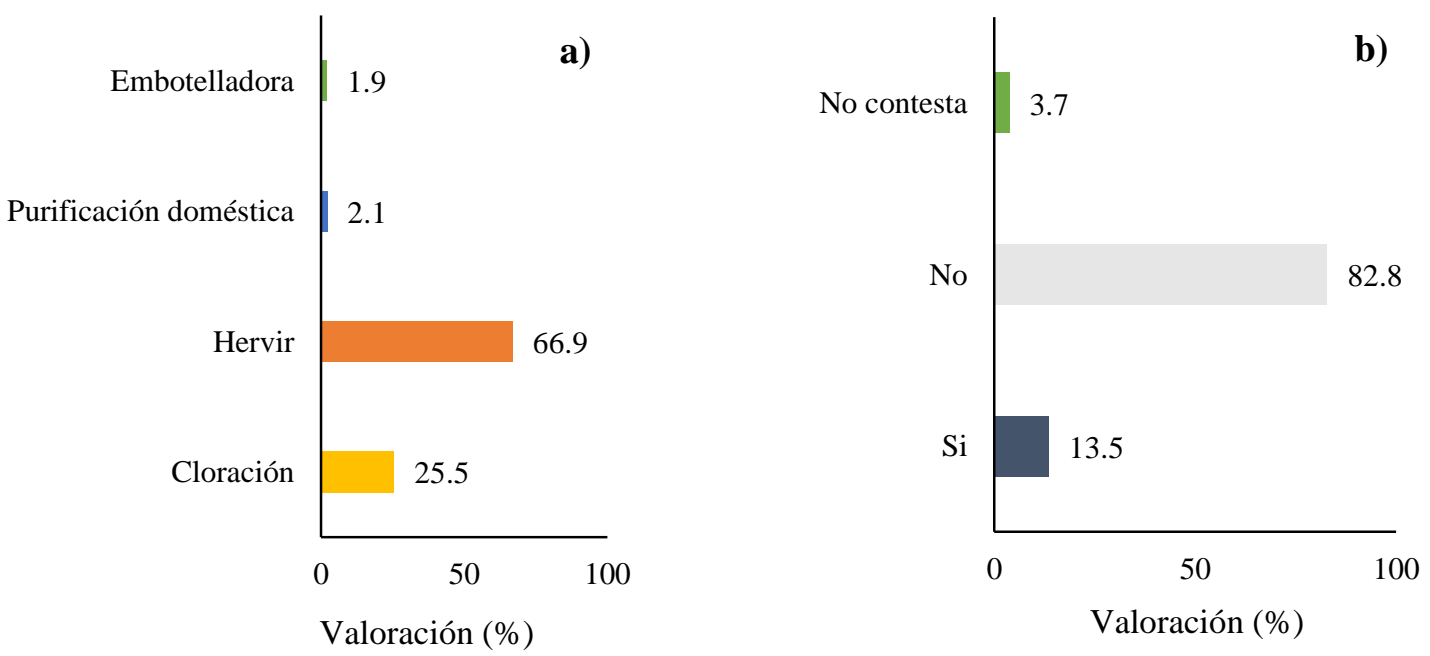

\section{CONCLUSIONES}

Los resultados encontrados demostraron que el 59, $4 \%$ de los hogares del municipio de Quevedo tienen acceso agua potable. Sin embargo, se detectó que el 36,5 y 16,7 \% de las viviendas adquieren agua con mal sabor y olor. Adicionalmente, se encontró que en el 
$55,9 \%$ de los hogares sus habitantes han presentado síntomas de diarreas por el consumo de agua, seguido de 13,8; 9,1 y 8,8 \%. de hogares con vómitos, cólicos y dolores abdominales. Problemas de salud asociado por la calidad del abastecimiento hídrico que tienen los hogares y el poco conocimiento que tienen los habitantes en técnicas de purificación del agua.

\section{REFERENCIAS BIBLIOGRAFICAS}

Bracho-Fernández, Irguin A., Fernández-Rodríguez, Moraima. (2017). Evaluación de la calidad de las aguas para consumo humano en la comunidad venezolana de San Valentín, Maracaibo. Minería y Geología 33(3), 341-352.

Brossard Peña, E., Gafas González, C., Hernández Meléndrez, D. E. Figueredo Villa, K. (2020). Enfermedades de transmisión hídricas en el cantón Penipe, Ecuador 20162017

Castro, M., Almeida, J., Ferrer, J., and D. Diaz. (2014). "Water Quality Indexes: Evolution and Trends at the Global Level", ing. Solidar. 10 (17): 111-124.

Committee on Environmental Health y Committee on Infectious Diseases ediatrics.2009. Consumo de agua de pozos particulares y riesgos para los niños. Pediatrics (Ed esp). 67(6):363-369.

Dalin, C. Konar, M., Hanasaki, N., Rinaldo, A., Rodríguez-Iturbe I. (2012). Evolución de la red mundial de comercio de agua virtual Proc Natl Acad Sci , 109 ( 16 ): 5989 $-5994$.

Domínguez Serrano, Judith. (2010). El acceso al agua y saneamiento: Un problema de capacidad institucional local. Análisis en el estado de Veracruz. Gestión y política pública, 19(2), 311-350.

Fernández Cirelli, Alicia (2012). El agua: un recurso esencial. Química Viva, 11(3),147170.

García Sánchez, M. R. Godínez Alarcón, G., Pineda Avonza, B., Reyes Añorve, J. (2015). Derecho al agua y calidad de vida. RIDE Revista Iberoamericana para la Investigación y el Desarrollo Educativo, 6(11).

Guzmán, Blanca L., Nava, G., Díaz, P. (2015). La calidad del agua para consumo humano y su asociación con la morbimortalidad en Colombia, 2008-2012. Biomédica, $35(2), 177-190$ 
Instituto Nacional de Estadística y Censo Agropecuario (INEC). (2016). Indicadores ODS de agua, saneamiento e higiene en Ecuador. 116p.

Instituto Nacional de Estadística y Censo Agropecuario (INEC). (2017). Medición de los indicadores ODS de Agua, Saneamiento e Higiene (ASH) en el Ecuador. Ecuador: Instituto nacional de estadistica y censos. Obtenido de https://www.ecuadorencifras.gob.ec/documentos/webinec/EMPLEO/2017/Indica dores\%20ODS\%20Agua, $\% 20$ Saneamiento $\% 20 \mathrm{e} \% 2$

OHigiene/Presentacion_Agua_2017_05.pdf

Mahlknecht, J., y Pastén Z. (2013). Diagnóstico de los recursos hídricos en América Latina Pearson México.

Ministerio de salud. (2021). Enfermedades transmitidas por agua y alimentos otras intoxicaciones alimentarias. Recuperado en: https://www.salud.gob.ec/enfermedades-transmitidas-por-agua-o-alimentos/.

Mite, F., Carrillo, M., y Durango W. (2010). Avances del monitoreo de presencia de cadmio en almendras de cacao, suelos y aguas en Ecuador. XII Congreso Ecuatoriano de la Ciencia del Suelo.

Naciones Unidas Comisión económica para América Latina y el caribe (CEPAL). (2017) Anuario estadístico de América Latina y el Caribe, Santiago, Chile.

Organización de las Naciones Unidas para la Agricultura y la Alimentación. (FAO) (2018). Sistema de información de la FAO sobre agua y agricultura Italia, Roma.

Ramírez, H.; Jaramillo, J. (2015). Agentes naturales como alternativa para el tratamiento del agua. Rev . Fac . Cienc . Básicas (Colombia). 11(2):136-153. 